\title{
Genomic signatures of flax diversification and improvement
}

\author{
Duk M. ${ }^{1}$, Kanapin A. ${ }^{1}$, Samsonova A. ${ }^{1}$, Rozhmina T. ${ }^{2}$, Samsonova M. ${ }^{1}$ \\ ${ }^{1}$ Peter the Great St. Petersburg Polytechnic University, St. Petersburg, Russia \\ ${ }^{2}$ Federal Research Centre for Bast Fiber Crops, Torzhok, Russia \\ *email: m.samsonova@spbstu.ru
}

Flax is a founder crop domesticated by humans since Neolithic times. Flax cultivation spread from the Fertile Crescent into Indian subcontinent, Central Asia, Mediterannean and East Africa, thus forming centers of secondary diversity (Vavilov, 1926, 1951), where the crop management practices were adapted to specific geographic regions and patterns of use. Through these processes two main subgroups of the crop appeared, namely fiber flax and oil flax. By the end of the XIX century northern regions of Russia became major flax production centres, where spontaneous selection carried out by local peasantry led to the development of local landraces (i.e. kryazhs).

In this study we present new insights into genetic diversity of 306 flax accessions from collection of the Federal Research Center for Bast Fiber Crops, encompassing landraces, elite cultivars and breeding lines.

Our results demonstrate that the population structure of flax recapitulates the crop subdivision into subgroups, as well as into landraces and cultivars. Signatures of gene flow in kryazh landraces were detected from both landraces originated to the West (Mediteranean, West Asia, Caucasus, Egypt) and to the East (Central Asia, Pakistan, North China) of Caspian sea. These findings challenge the hypothesis regarding kryazh landraces Central Asian origin (Zhykovsky, 1971). Genomic variation in plants attributed to the centers of secondary diversity correlates with geography and climate features. Regions with negative Tajima $\mathrm{D}$ values were found on most chromosomes in elite cultivars and breeding lines suggesting strong selection accompanying the crop improvement history. A comparison of regions of reduced diversity (e.g. high $\mathrm{F}_{\mathrm{st}}$ ) between krjazh landraces and cultivars pinpointed genomic loci under selection, which are presumably associated with the most recent breeding efforts of fiber flax. Furthermore, by thorough analysis of cultivars we reconstructed the fiber flax breeding history.

Acknowledgments: This work was supoorted by RSF fund, grant 19-16-00030.

60 PlantGen2021 